\title{
A tune of triumph
}

\author{
$\left(\frac{2}{2}\right)$ \\ Michael Rahbek Schmidt*, MD, PhD, DMSC; Niels Vejlstrup, MD, PhD \\ Department of Cardiology, Rigshospitalet, Copenhagen, Denmark
}

Right ventricular outflow tract (RVOT) and pulmonary valve malformations occur in approximately one fifth of babies with congenital heart disease, including tetralogy of Fallot, pulmonary atresia, and truncus arteriosus. The overall aim of the treatment of these conditions is to separate the ventricles and secure a stable unobstructed connection with preserved valve function between the right ventricle and the pulmonary arteries, frequently through a series of interventions during the growth of the child. This typically involves early palliation with either a modified Blalock-Taussig shunt between subclavian and pulmonary arteries, or a transannular patch of the RVOT and pulmonary valve, followed by the insertion of a valved right ventricle to pulmonary artery (RV-PA) conduit. Similar conduits are also used in the Ross procedure for aortic stenosis. RV-PA conduits, whether synthetic, xenografts or homografts, have a limited lifespan due to progressive calcification and deterioration, as well as the fact that children may outgrow them. Consequently, a large proportion of patients with RV-PA conduits will develop increasing obstruction or regurgitation inducing pressure and/or volume overloading of the right ventricle. Although this condition can usually be tolerated for some years, RV dysfunction and related symptoms will eventually occur, requiring further intervention to relieve obstruction or eliminate regurgitation.

Since Philipp Bonhoeffer launched the Melody ${ }^{\circledR}$ valve (Medtronic, Minneapolis, MN, USA) for percutaneous pulmonary valve implantation (PPVI) to prolong the longevity of RV-PA conduits, the treatment options have changed radically. The Melody valve was the first transcatheter heart valve to be implanted in humans (2000) and the first to be commercially available (2006), and thus preceded the clinical use of transcatheter aortic valve implantation (TAVI), although both of the valve types were invented in the mid 1990s. In several ways, the early clinical adoption of the Melody valve has helped to pave the way for the subsequent huge clinical and commercial success of TAVI, and follow-up data from early implantations keep providing important information regarding valve longevity and clinical outcomes that are also relevant in the wider context of TAVI.

In the current issue of EuroIntervention, Cools et al present one of the largest studies to date of long-term outcome after PPVI with the Melody valve'.

Article, see page 988 
The non-randomised observational study includes 185 patients up to 11 years after the PPVI procedure and provides novel and important information. The Belgian group is a leading PPVI unit, and their reported data on haemodynamic performance and infective endocarditis (IE) incidence are thus likely to be at least on a par with most other centres. The observed relatively stable gradients and surprisingly low frequency of significant regurgitation over more than five years provide reason for optimism regarding longevity of the Melody valve and demonstrate a need for reintervention lower than that which has been reported for surgical conduits. However, although stable, the antegrade gradients are not zero $(36 \pm 12 \mathrm{mmHg}$ after 10 years), and the RV-PA obstruction will entail a chronic pressure load on the RV with risk of fibrosis, dysfunction, arrhythmia, etc.

More worrying to most clinicians is the concern for IE associated with the Melody valve. The reported incidence of IE varies between studies, but the most important finding in the present study may be the apparently substantial risk-reducing effect of strict adherence to ESC endocarditis prophylaxis guidelines. In this cohort, the risk of IE was reduced to a mere $1 \%$ /year with the non-deviation strategy but this required rigorous hygienic measures as described by the authors, who also stress the importance of conveying this information to parents, general practitioners, and dentists. The specific challenges related to dealing with such precautions in younger patients should not be neglected.

Interestingly, the authors have refrained from the use of antiplatelet therapy as part of the IE prophylaxis. While it may raise eyebrows among some clinicians, this approach - substantiated by the findings in the current study - is not unreasonable, given the relevant point made, that IE prophylaxis through prevention of microthrombi is based on speculation and lacks evidence.

Overall, PPVI with the Melody valve is safe and durable when used in the right patients and with diligent focus on IE prevention. While surgical conduits may still be preferable in some countries due to the cost of transcatheter heart valves or absence of PPVI operators, the Melody valve experience has been a game changer towards percutaneous approach for valve replacement with a positive impact far beyond the Fallot patients with stenosed homografts for whom it was first intended.

\section{The melody of the future}

How can we ensure that PPVI becomes a lasting success? A key factor will be sustained focus on refinement and development. Several factors indicate that the valve and PPVI procedure can be optimised further:

- Some studies suggest that endocarditis is less frequent when the XT generation of the SAPIEN valves (Edwards Lifesciences, Irvine, CA, USA) is used for $\mathrm{PPVI}^{2,3}$, although these data were based on high endocarditis incidence in the population treated with Melody valves ${ }^{4,5}$ (Table 1). Further studies into the reason for this difference (if present), e.g., comparison of preparation techniques, microstructures of the leaflets, residual pressure gradient, etc., could shed light on areas with potential for improvement. Furthermore, since vegetations inside a stent valve may be difficult to visualise by transthoracic and transoesophageal echocardiography, other imaging modalities such as intracardiac echocardiography and 18F-FDG PET/CT scan may be required in order to make a definite IE diagnosis.

- While the no-nonsense simplicity of the Melody valve and the Ensemble ${ }^{\circledR}$ delivery system (Medtronic) is a blessing for new operators because it is easy to understand and learn to use, it remains a somewhat old-fashioned bundle. Development has essentially halted while TAVI systems are currently on their third generation, and finger crimping and non-steerable sheaths are unlikely to be the way ahead.

- Prevention of stent fracture by pre-stenting may seem like an obsolete technique, but it creates a stable scaffold for the subsequent PPVI and has so far become widely accepted as the standard. The balance between creating a flexible system allowing the valve to move through the RV and building a strong stent able to withstand movement and radial compression is complex. Possibly the current two-step method is here to stay as it offers

Table 1. Endocarditis after percutaneous pulmonary valve implantation with the Melody valve.

\begin{tabular}{|c|c|c|c|c|}
\hline & Cools et al ${ }^{1}$ & Hascoet et $\mathrm{al}^{3}$ & $\begin{array}{l}\text { McElhinney } \\
\text { et al } \text { a }^{* * 4}\end{array}$ & $\begin{array}{c}\text { Malekzadeh-Milani } \\
\text { et } \mathrm{a}^{5}\end{array}$ \\
\hline Number of patients & 185 & 32 & 311 & 85 \\
\hline Patient age & $19.4 \pm 13.2$ & $19.8(15.8-28.9)$ & $\mathrm{N} / \mathrm{A}$ & $23.3 \pm 10.5$ \\
\hline Study period & $2006-2017$ & $2008-2016$ & $2007-2012$ & $2009-2012$ \\
\hline Median follow-up time (years) & Mean 4.4 , up to 11 & 4.9 & 2.5 & 2 \\
\hline Systolic RV-PA gradient post-implantation (mmHg) & $23.4 \pm 11.1$ & N/A & N/A & $12.4 \pm 7.4$ \\
\hline Proportion of patched/native RVOT (\%) & 32.8 & 16.5 & 0 & 0 \\
\hline Proportion of patients on aspirin post-implantation (\%) & 0 & $100(6 \mathrm{mo})$ & N/A & 91 \\
\hline Endocarditis in Melody valves (\%/person years) & $2.3^{*}$ & $5.7(2.9-11.4)^{* *}$ & 2.4 & 3 \\
\hline Repeat pulmonary valve replacement during follow-up (\%) & 22 after 10 years & 15.5 after 3.4 years & $\mathrm{N} / \mathrm{A}$ & $\mathrm{N} / \mathrm{A}$ \\
\hline
\end{tabular}


several advantages, but new materials and modern engineering may provide novel solutions. Self-expanding straight or hourglass-shaped stents may also widen the applicability of PPVI in native or patch-augmented outflow tracts.

- The use of 2D and 3D computed tomography (CT) has become widely adopted among TAVI operators to plan procedures in regard to vascular access, wire/sheath positioning, optimised fluoroscopy angles and exact placement of the valve. Overlay of echo and CT images is increasingly offered by X-ray system manufacturers. These modern multimodality imaging techniques will most likely also become more prevalent in PPVI.

In conclusion, PPVI is a great success story, which is evidenced by the current paper by Cools and co-authors. Clinical outcomes are good, valve longevity and freedom from reintervention high, long-term haemodynamic results encouraging, stent fractures virtually eliminated by pre-stenting techniques, and endocarditis - though still a major concern - often medically treatable, rarely lethal and to a large extent avoidable by stringent prophylactic measures. Compared to surgical conduit procedures, which also involve several potential complications (including risk of redo surgery, somatic outgrowth, anastomotic stenosis of the conduit, valvular stenosis, conduit kinking, sternal compression, intimal proliferation, conduit calcification, and aneurysmal degeneration), PPVI seems at least non-inferior and far less traumatic for the patient.

\section{Conflict of interest statement}

The authors have no conflicts of interest to declare.

\section{References}

1. Cools B, Brown S, Budts W, Heying R, Troost E, Boshoff D, Eyskens B, Gewillig M. Up to 11 years of experience with the Melody valved stent in the right ventricular outflow tract. EuroIntervention. 2018;14:e988-94.

2. Wilson WM, Benson LN, Osten MD, Shah A, Horlick EM. Transcatheter Pulmonary Valve Replacement With the Edwards Sapien System: The Toronto Experience. JACC Cardiovasc Interv. 2015;8:1819-27.

3. Hascoet S, Mauri L, Claude C, Fournier E, Lourtet J, Riou JY, Brenot P, Petit J. Infective Endocarditis Risk After Percutaneous Pulmonary Valve Implantation With the Melody and Sapien Valves. JACC Cardiovasc Interv. 2017;10:510-7.

4. McElhinney DB, Benson LN, Eicken A, Kreutzer J, Padera RF, Zahn EM. Infective endocarditis after transcatheter pulmonary valve replacement using the Melody valve: combined results of 3 prospective North American and European studies. Circ Cardiovasc Interv. 2013;6:292-300.

5. Malekzadeh-Milani S, Ladouceur M, Patel M, Boughenou FM, Iserin L, Bonnet D, Boudjemline Y. Incidence and predictors of Melody ${ }^{\circledR}$ valve endocarditis: a prospective study. Arch Cardiovasc Dis. 2015;108:97-106. 